\section{Recherche sur}

les cellules souches

embryonnaires importées

\author{
Avis consultatif $n^{\circ} 1 / 2001^{*}$ \\ CNE Commission nationale d'éthique pour la médecine humaine
}

\begin{abstract}
Remarque préalable
La fonction de la Commission nationale d'éthique pour la médecine humaine (CNE) est de nature consultative. La Commission ne peut pas prendre de décision, dans quelque domaine que ce soit, à la place des instances qu'elle conseille. Son rôle consiste plutôt à souligner les aspects d'ordre éthique qui, à son avis, sont importants et dont on doit tenir compte lorsque une décision doit être prise dans un contexte donné. Elle présente également les résultats auxquels on peut s'attendre selon la décision prise par le mandataire. L'avis consultatif résulte d'un vote à la majorité simple et, le cas échéant, les points de vue de la minorité et de la majorité y sont présentés.

La question de l'importation en Suisse de cellules souches embryonnaires humaines à des fins de recherche médicale a soulevé dans le pays un sentiment de malaise, de nature généralement mal définie. Le travail de la CNE a consisté, d'un point de vue éthique, à préciser la nature de ce malaise, à en détecter les raisons et à les clarifier, pour les rendre accessibles à la critique et pour en vérifier la validité.
\end{abstract}

\section{Exposé du problème}

A la suite d'une demande de fonds, la question a été posée de savoir s'il existait des réserves de nature éthique quant au financement par les pouvoirs publics de la recherche sur les cellules souches embryonnaires importées de l'étranger (USA). La production de ces cellules présuppose la destruction

* Après la séance plénière du 31 août 2001, le texte a été rectifié lors d'une procédure écrite et autorisé le 19 septembre 2001.

Correspondance:

Prof. Dr. phil. Christoph Rehmann-Sutter

Président NEK-CNE

c/o Office fédéral de la santé publique

CH-3003 Berne d'embryons au stade de blastocystes. Des cellules souches embryonnaires peuvent être extraites de la masse cellulaire interne de ces blastocystes, pour être ensuite multipliées en culture. En Suisse, la situation juridique n'est pas claire en ce qui concerne ce processus de production. Depuis le printemps 2000, une demande d'autorisation d'un groupe de recherche de Genève est examinée au Fonds national suisse pour la recherche scientifique. Cette demande prévoit l'étude de cellules souches embryonnaires importées. Dans le cadre de ce projet, les chercheurs ont l'intention d'essayer d'extraire in vitro des myocytes du cœur (cellules musculaires cardiaques) et des cellules souches hématopoïétiques à partir de cellules souches embryonnaires.

Lors de sa séance du 13 juin 2001, la commission $\mathrm{du}$ Conseil national de la recherche scientifique, compétente pour accorder les subventions attribuées par le Fonds national, a décidé de ne pas financer le projet genevois pour l'instant. Cette demande, auparavant, avait été considérée comme valable d'un point de vue scientifique, donc recevable en vue d'un financement par cette instance. Deux rapports d'expertise ont été demandés, respectivement aux commissions d'éthique de l'Académie Suisse des Sciences Médicales (ASSM) et de l'Université de Genève. Ces commissions ont émis un jugement positif sur ce projet. Une expertise juridique supplémentaire a conclu que l'importation de cellules souches embryonnaires, prévue dans le projet de recherche, n'enfreignait pas la législation suisse. Selon la communication du Fonds national aux médias de ce même 13 juin 2001, l'ajournement décidé par le FNS était motivé par le désir de ne pas prendre une position préalable à une discussion politique sur les aspects éthiques et juridiques du projet. Une décision définitive a été toutefois annoncée pour «au plus tôt la fin de l'été/ l'automne de cette année», aussi bien en ce qui concernait la demande de subventions déposée que sur une prise de position fondamentale du Fonds national à l'égard de tous les projets de recherche dont les auteurs prévoient d'utiliser des cellules souches embryonnaires humaines importées.

Peu après la mise en place de la CNE par le Conseil fédéral (début juillet 2001), des prises de contact avec les responsables principaux du Fonds national ont montré qu'un appréciation de la CNE serait utile et bienvenue dans cette affaire. Ainsi, le Fonds national a mis une documentation détaillée à la disposition de la CNE, afin qu'elle puisse émettre un avis sur cette question. Ainsi, l'avis de la CNE peut être encore intégré dans le processus de décision en cours au sein du Fonds national.

Lors de ces derniers mois, la presse a fait preuve d'un intérêt accru à l'égard de la recherche sur les embryons, reflétant en cela une sensibilité du public par rapport à l'aspect éthique de ces questions. Cette prise de conscience du public s'est encore intensifiée en Suisse, en raison de ces discussions sur les subventions à accorder ou non à la recherche sur les cellules souches embryonnaires importées. Cet intérêt a été particulièrement renforcé par la décision (en mai 
2001) de l'Association allemande pour la recherche scientifique de subventionner la recherche sur des cellules souches embryonnaires importées, un arrêté qui a provoqué par la suite de multiples controverses. On peut noter également l'importance de la décision du président américain George $W$. Bush, au début du mois d'août 2001, laquelle permettait le soutien, au moyen de fonds publics, de recherches pratiquées sur des cultures de cellules souches embryonnaires humaines déjà existantes, sans que toutefois la production de nouvelles cultures à partir d'embryons créés à cet effet ne soit autorisée.

Dans la presse suisse, certains commentateurs se sont exprimés de telle manière que l'on a pu avoir l'impression que la législation en vigueur en Suisse interdisait la production de cellules souches embryonnaires, ce qui n'est à l'évidence pas le cas lorsque cette production est effectuée en vue d'un projet parental. Comme on a pu le lire bien souvent, cette interdiction s'étendrait même à l'utilisation d'embryons qui auraient dû être détruits de toute façon, puisqu'ils avaient été considérés par les parents concernés comme "surnuméraires», c'est-à-dire inutiles dans l'optique d'un projet de procréation médicalement assistée. Toutefois, si une telle production d'embryon était interdite à l'intérieur des frontières nationales et/ou considérée comme condamnable, le fait d'encourager, ou simplement de permettre, l'importation de telles cellules provenant de l'étranger constituerait une contradiction évidente d'un point de vue moral. On peut envisager une situation similaire si l'on importait en Suisse le butin provenant d'un vol commis dans un pays où ce vol ne serait pas frappé d'interdit: une démonstration parfaite d'un moral à double facette! Avec une telle toile de fond, il serait difficile de justifier, aux yeux du public, une décision du Fonds national en faveur des chercheurs.

\section{Considérations}

Contrairement à une opinion très répandue, le statut juridique, en Suisse, des cellules souches embryonnaires produites à partir de blastocystes surnuméraires après des fécondations in vitro et qui doivent être détruites, n'est pas clair, au moins au yeux de la CNE. L'article 5 § 3 de la loi sur la procréation médicalement assistée, qui interdit le prélèvement de cellules embryonnaires in vitro à des fins d'examen, vise clairement le diagnostic préimplantatoire. Le texte en lui-même, il est vrai, se réfère tout de même à la recherche sur l'embryon. Toutefois, si l'on tient compte de l'intention du législateur et du contexte de cette norme juridique (il s'agit systématiquement de techniques employées dans la médecine de procréation), il en découle certes une interdiction du diagnostic préimplantatoire, mais pas une interdiction de l'extraction de cellules souches embryonnaires à partir d'embryons "surnuméraires». Un éclaircissement de ce point pourrait être apporté par la voie législative (normes juridiques complémentaires, par ex. dans le cadre du projet de loi "Recherche sur l'être humain»).
L'aspect éthique du problème, à savoir si, dans certaines conditions, il pourrait être admis d'utiliser les embryons dits "surnuméraires" provenant de fécondations in vitro, qui doivent être détruits de toute façon, pour la production de cellules souches embryonnaires, est encore débattu et la question n'a pas encore été tranchée en Suisse. La multiplicité des réponses apportées à cette question est en relation, jusqu'à un certain point, avec la variété des points de vue sur le statut moral de l'embryon. En outre, il importe d'évaluer spécifiquement la recherche sur les cellules souches embryonnaires, notamment sa nécessité, ses méthodes et ses objectifs. Ce débat particulier sur la recherche sur les cellules souches embryonnaires n'a pas suffisamment eu lieu dans le domaine public. Dans le secteur de l'éthique, quelques comités importants ont déjà partiellement pris position à cet égard. La CNE, quant à elle, ne s'est pas encore déterminée dans ce domaine.

Nous allons assister en Suisse à une période transitoire, jusqu'à ce que ces questions soient tranchées, tant quant à leur aspect légal que moral. Pendant cette période de transition, l'instauration d'actions ou de décisions portant préjudice à la recherche serait très néfaste. Afin de ne pas porter atteinte à la qualité des réflexions nécessaires à déterminer une attitude claire dans notre pays, il est important de préserver la réversibilité des actions et des décisions qui seraient prises, dans un sens comme dans l'autre, soit dans la direction d'une autorisation, ou vers celle d'une interdiction. Il est toutefois possible, dans le même temps, de faire avancer activement les réflexions dans ce domaine.

\section{Appréciation}

La CNE s'inquiète de ce que le fait d'accéder à la demande qui prévoit l'importation de cellules souches embryonnaires dans un but de recherche conduise à des décisions dommageables dans la situation qui règne actuellement en Suisse. Il lui tient particulièrement à cœur que des décisions ne soient prises qui se répercuteraient ensuite en créant des conditions irrévocables lors de la période d'examen de cette question, autant du point de vue juridique qu'éthique. Le même risque existe toutefois dans le sens opposé, à savoir qu'un rejet de la demande de subside soit interprété comme une décision du Fonds national contre la recherche sur les cellules souches embryonnaires. C'est pourquoi, aucune situation irréversible ne doit être créée.

Pour cette raison, une majorité des membres de la commission conseille de mettre en attente les demandes de subside prévoyant l'importation de cellules souches embryonnaires, jusqu'à ce qu'une mise au point soit achevée, tant du point de vue juridique qu'éthique. Une minorité considère néanmoins que la situation peut rester réversible même dans le cas d'un accès à une demande de recherche dans ce domaine.

En tout cas, qu'une demande de subside dans ce domaine soit acceptée, refusée ou ajournée, il importe 
d'exprimer clairement qu'il s'agit là d'une décision ponctuelle ne pouvant faire jurisprudence en Suisse.

La CNE souligne clairement l'importance de faire évoluer activement le débat sur la légitimité de l'emploi de cellules souches embryonnaires, importées ou non, en Suisse, tant du point de vue juridique qu'éthique. Elle tient à encourager ce débat grâce à une contribution spécifique. Pour cette raison, elle va placer le problème éthique fondamental de la légitimité de la recherche sur les cellules souches embryonnaires au centre de son activité dans les temps à venir.

Certains membres de la commission soulignent l'aspect commercial douteux de l'acquisition des cellules souches. Les conditions imposées par certains pourvoyeurs étrangers, portant sur la livraison et l'utilisation de leurs lignées de cellules, ressemblent fort, sur le fond, à une relation commerciale, même si elles ne peuvent être considérées comme équivalentes à un "commerce" au sens strict de la loi. Les indemnités pour frais d'administration, les redevances d'exploitation de licence etc. contribuent à l'interpénétration de la recherche avec des intérêts économiques.

\section{Exposé des motifs}

La question de savoir s'il est admissible d'utiliser des embryons humains pour des buts de recherche et si oui, dans quelles conditions, est étroitement liée à l'opinion fondamentale qu'à l'homme sur la valeur d'une vie humaine en devenir. Il s'agit de préciser concrètement si la recherche embryonnaire est fondamentalement justifiable et, si cela est le cas, dans quelles conditions et dans quelles limites. Ces questions sont de l'ordre de la perception morale et de l'examen des valeurs éthiques. Elles doivent trouver une réponse lors d'une discussion éthique ouverte et lors d'un débat sur les aspects juridiques correspondants. Les réponses ne peuvent pas provenir exclusivement de la communauté scientifique et des organismes de subvention.

Ce qui est en jeu ici dépasse les limites d'une autorisation accordée aux demandes de subsides en vue de projets de recherche qui ont été déposées actuellement. Il s'agit de déterminer dans quelle(s) limite(s) la vie humaine en devenir peut, ou non, être mise à disposition des chercheurs. De la détermination de ces limites, d'autre part, dépend la création et l'établissement d'un nouveau domaine de recherche en Suisse et le développement de nouvelles possibilités de retombées biomédicales, connues sous le nom de médecine régénératrice. Un examen attentif de l'évolution de cette question au niveau de la population suisse est important, parce qu'il est dans l'intérêt (un intérêt réfléchi) du développement de la médecine, ainsi qu'en faveur d'une amélioration de la relation entre les chercheurs et le public. Ce public pourrait percevoir l'instauration de décisions en faveur de l'importation de cellules souches embryonnaires comme un acte de pouvoir médical. D'un point de vue éthique, cette situation pourrait être embarrassante, dans la mesure où elle ne concernerait pas seulement la recherche, mais où elle toucherait également à ses conditions d'exercice. Comme ce sujet donne lieu à de vives controverses, aussi bien en Suisse qu'à l'étranger, et qu'une décision ne peut être prise qu'après un examen exhaustif de tous les arguments disponibles, l'acceptation ou le rejet de demandes de subsides de recherche dans ce domaine semblerait équivaloir à une décision qui pourrait faire jurisprudence et bloquer la situation.

Comparés à la question de la légitimité éthique de l'utilisation à des buts de recherche de cellules souches embryonnaires humaines et aux raisons qui plaident en sa faveur, les questions relatives à l'interprétation de la loi et à l'importation de cellules souches de l'étranger revêtent une importance secondaire et pour ainsi dire contextuelle. Les décisions qui devront être prises auront à suivre un ordre de priorité à même de refléter la hiérarchie selon laquelle doivent s'organiser ces mêmes questions: ceci constitue la condition essentielle de l'impartialité des discussions qui se tiendront au sein de notre commission.

Dans certaines situations critiques menaçant gravement la santé, ou même la vie, de certains patients, il pourrait être justifié d'anticiper l'importation de cellules souches embryonnaires extraites à l'étranger, avant d'avoir éclairci les questions éthiques et juridiques qui se posent en Suisse. Il faudrait alors assumer le risque des effets d'une telle décision, à savoir l'altération des conditions nécessaires à un débat serein et objectif dans notre pays. Une telle situation critique, toutefois, n'est pas véritablement présente dans la demande de subside au Fonds national actuellement discutée. Il n'existe pas de relation directe entre le projet actuel de recherche genevois et la nécessité de sauver des vies humaines. Il existe certes l'espoir que ce genre de recherche contribuera à guérir des maladies et à sauver des vies humaines, mais cet espoir ne peut actuellement être concrétisé au moyen du projet en discussion. Lorsqu'il s'agira de peser le pour et le contre de (l'acceptation ou du refus de) l'autorisation d'importation de cellules souches embryonnaires, il faudra donc inclure les retombées médicales comme une potentialité pour le long terme.

\section{Commentaire}

Cet avis consultatif ne condamne pas la recherche sur les cellules souches embryonnaires. Il est reconnu que la recherche sur et avec des cellules souches embryonnaires représente un secteur prometteur, tant du point de vue scientifique que thérapeutique. Une évaluation, du point de vue de l'éthique, de ce type de projet de recherche dépend de la question centrale de savoir si, et dans quelles conditions, des embryons au stade de blastocystes peuvent être utilisés en vue de l'extraction de ces cellules souches. Ce n'est en effet qu'alors que ce domaine de recherche dont nous débattons actuellement, pourra être effectué sur les 
embryons. Il ne s'agit pas ici, en effet, d'un simple protocole de recherche observationnel portant sur la description du comportement naturel de l'embryon, son intégrité demeurant inviolée, mais bien de l'extraction de certaines de ses cellules et de leur culture en dehors de leur enveloppe organique. Lors de cette opération, l'embryon est détruit. Cette procédure représente une question fondamentale d'éthique est elle est considérée comme prioritaire par la CNE dans son travail pour un proche avenir. Une autre question, étroitement liée à celle qui nous occupe, doit également être posée, celle de savoir s'il est établi clairement que les mêmes objectifs que ceux qui sont poursuivis avec l'emploi de cellules souches embryonnaires ne peuvent pas être atteints avec des cellules souches adultes.

La question de l'importation des cellules souches embryonnaires s'est toutefois posée à la CNE indépendamment de la demande genevoise de subside au FNS. Cette question s'est ainsi posée parce que de telles lignées de cellules ont déjà été extraites de nos jours, qu'elles existent donc, et qu'on peut les commander à différents fournisseurs. Utiliser ces cellules souches pour la recherche ne signifie pas qu'il faille les extraire d'embryons, cette extraction ayant déjà eu lieu préalablement à leur mise en culture. Selon le registre actuel du "National Institut of Health" (NIH), les chercheurs peuvent se procurer ces cellules dans de nombreux centres aux USA, en Suède, en Australie, en Inde et en Israël, dans différentes conditions contractuelles. Leur extraction a eu lieu, et a lieu aujourd'hui dans différents systèmes juridiques et au sein de différentes traditions morales. L'exploration des caractéristiques des cellules embryonnaires et leur utilisation en tant qu'outils scientifiques est de fait possible actuellement sans qu'il faille utiliser de nouveaux embryons pour leur extraction.

Les scientifiques, il est vrai, se demandent s'il existe une quantité suffisante de ces lignées et si leur qualité peut se maintenir sans que l'on procède à l'extraction de nouvelles lignées à partir d'embryons. Il est possible que, pour des raisons d'ordre scientifique et médical, mais aussi politique et même éthique, il soit indiqué de ne pas se contenter des lignées de cellules déjà existantes et de promouvoir en Suisse également, dans certaines conditions et dans un environnement contrôlé, l'extraction de nouvelles lignées de cellules à partir d'embryons "surnuméraires" issus de fécondations in vitro, et qui ont déjà été détruits. L'avis consultatif présent ne doit pas anticiper cette réflexion, qui est elle-même importante et urgente.
Si, dans tous les pays, les chercheurs qui travaillent dans le domaine public se comportent de manière réservée, pour des motifs évidents, à l'égard de la production de cellules souches embryonnaires humaines, cette attitude risque d'entraîner la formation de niches économiques sur le marché, qui ne manqueront pas d'être saisis par l'industrie privée. Le Fonds national suisse devrait prendre des dispositions pour que l'utilisation de cellules souches importées ne rende pas les chercheurs suisses dépendants des fournisseurs de cellules souches avec des intérêts commerciaux. Cet aspect est d'importance pour l'avis consultatif définitif de la Commission. Il constitue de fait un argument en faveur de la production en Suisse, dans les meilleurs conditions possibles, de cellules souches, pour le cas où la recherche suisse désire et nécessite leur utilisation. En Suisse, en effet, l'embryon à son premier stade d'évolution ne sera pas traité comme une "chose» que l'on peut commercialiser librement.

Si la recherche sur les cellules souches embryonnaires débouche un jour sur des thérapies proposées à l'extérieur du pays, mais interdites en Suisse, on ne pourra pas empêcher les patientes et les patients de se rendre à l'étranger pour bénéficier de ces thérapies. Il en résulterait une situation contradictoire au point de vue moral. Cette contradiction pourrait être sans doute résolue par une promotion rapide de la recherche sur les cellules souches en Suisse, avec l'application de toutes les possibilités juridiques à notre disposition. La majorité de la CNE ne suit toutefois pas cette façon de voir. En effet, pour que la recherche dans ce domaine puisse atteindre une légitimité acceptée par la population, il importe de respecter une procédure décisionnelle impartiale et transparente. La recherche scientifique, quant à elle, agit de manière plus "opportuniste», ce qui est tout à fait compatible avec sa vocation propre. Ainsi, elle utilise toutes les voies qui s'ouvrent à elle en raison du progrès continuel du savoir et de la technique, sans référence systématique à l'opinion de la population. Une véritable légitimité, toutefois, ne peut être acquise par ces voies opportunistes. La seule existence d'une possibilité d'action n'équivaut en rien à sa légitimité éthique.

La politique de subvention du Fonds national n'a, il est vrai, aucune influence directe sur les promoteurs privés de la recherche (fondations, instituts). En raison du caractère de droit public du Fonds national, sa politique revêt toutefois une importance particulière pour le débat dans le cadre de la Suisse. Néanmoins, les arguments de la CNE présentés ici s'adressent fondamentalement à tous les chercheurs opérant en Suisse et à tous ceux qui les subventionnent. 